\title{
Complejo esclerosis tuberosa: Reporte de un caso clínico neonatal
}

\author{
Tuberous sclerosis complex: Report of a neonatal case
}

\author{
Patricia Desvars' ${ }^{1}$, Larissa Genes', Silvia Irala', Elvira Mendieta', Norma Astigarraga², Ramón Mir ${ }^{1}$
}

\section{RESUMEN}

El complejo de esclerosis tuberosa es una afección genética, aproximadamente dos tercios de los casos ocurren esporádicamente; se caracteriza por lesiones y tumores benignos (hamartomas) en múltiples sistemas orgánicos. La detección prenatal precisa es importante para el pronóstico, una decisión sobre el resultado del embarazo y el asesoramiento.

Se reporta el caso de una gestante sana y su feto con diagnóstico presuntivo de esclerosis tuberosa, por hallazgo de masas intracardiacas en ecografía prenatal, confirmados luego del nacimiento, con afectación de otros órganos, cumpliendo con criterios mayores de diagnóstico. El caso presentado es el primero registrado en el Departamento de Neonatología y tiene el objetivo de mostrar la aproximación diagnóstica prenatal y postnatal de una enfermedad relativamente rara.

Palabras claves: Esclerosis tuberosa, diagnóstico prenatal, rabdomioma cardiaco.

\section{INTRODUCCIÓN}

La esclerosis tuberosa es un desorden multisistémico, se cree que la penetrancia es completa, lo que dificulta el diagnóstico en algunas familias, particularmente en aquellas con rasgos sutiles ${ }^{(1)}$.

\begin{abstract}
Tuberous sclerosis complex is a genetic condition caused by mutations in the tumor suppressor genes TSC1 and TSC2, located on chromosomes 9 and 16 respectively. Approximately two-thirds of cases occur sporadically and the overall incidence has been estimated to be 1 in 5,800 live births. It is characterized by benign lesions and tumors (hamartomas) in multiple organ systems (brain, heart, skin, eyes, kidneys, lungs, liver). Accurate prenatal detection is important for prognosis, a decision about the pregnancy outcome, and counseling.

We report the case of a healthy pregnant woman and her fetus with a presumptive diagnosis of tuberous sclerosis, due to the finding of intracardiac masses on prenatal ultrasound, confirmed after birth, with involvement of other organs, fulfilling the major diagnostic criteria. The case presented is the first recorded case at our Department of Neonatology and this report illustrates the prenatal and postnatal diagnostic approach of a relatively rare disease.
\end{abstract}

Keywords: Tuberous sclerosis, prenatal diagnosis, cardiac rhabdomyoma.

Los criterios de diagnóstico se revisaron en 2013 para tener en cuenta el papel de las pruebas moleculares, donde una variante de TSC1 o TSC2 patógena puede servir como criterio independiente ${ }^{(1)}$.

\footnotetext{
${ }^{1}$ Universidad Nacional de Asunción. Facultad de Ciencias Médicas. Hospital de Clínicas. Departamento de Neonatología. San Lorenzo, Paraguay.

${ }^{2}$ Universidad Nacional de Asunción. Facultad de Ciencias Médicas. Hospital de Clínicas. Departamento de Cardiología Infantil. San Lorenzo, Paraguay.

Correspondencia: Patricia Desvars Correo: patydesro@hotmail.com

Conflicto de interés: Los autores declaran no poseer conflicto de interés.

Recibido: 17/09/2020 Aceptado:30/09/2020
}

Doi: https://doi.org/10.31698/ped.47032020009

(cc)BY Bste es un artículo publicado en acceso abierto bajo una Licencia Creative Commons CC-BY 4.0 
El diagnóstico clínico es un desafío, ya que la enfermedad es muy variable y sus manifestaciones continúan desarrollándose durante la vida de los individuos afectados ${ }^{(2)}$.

\section{DESCRIPCIÓN DEL CASO CLÍNICO}

Madre de 25 años de edad, primera gesta, controles prenatales suficientes, sin antecedentes patológicos personales ni familiares de valor. En la ecocardiografía fetal del segundo trimestre se observaron tumores compatibles con túberes. (Figura 1).
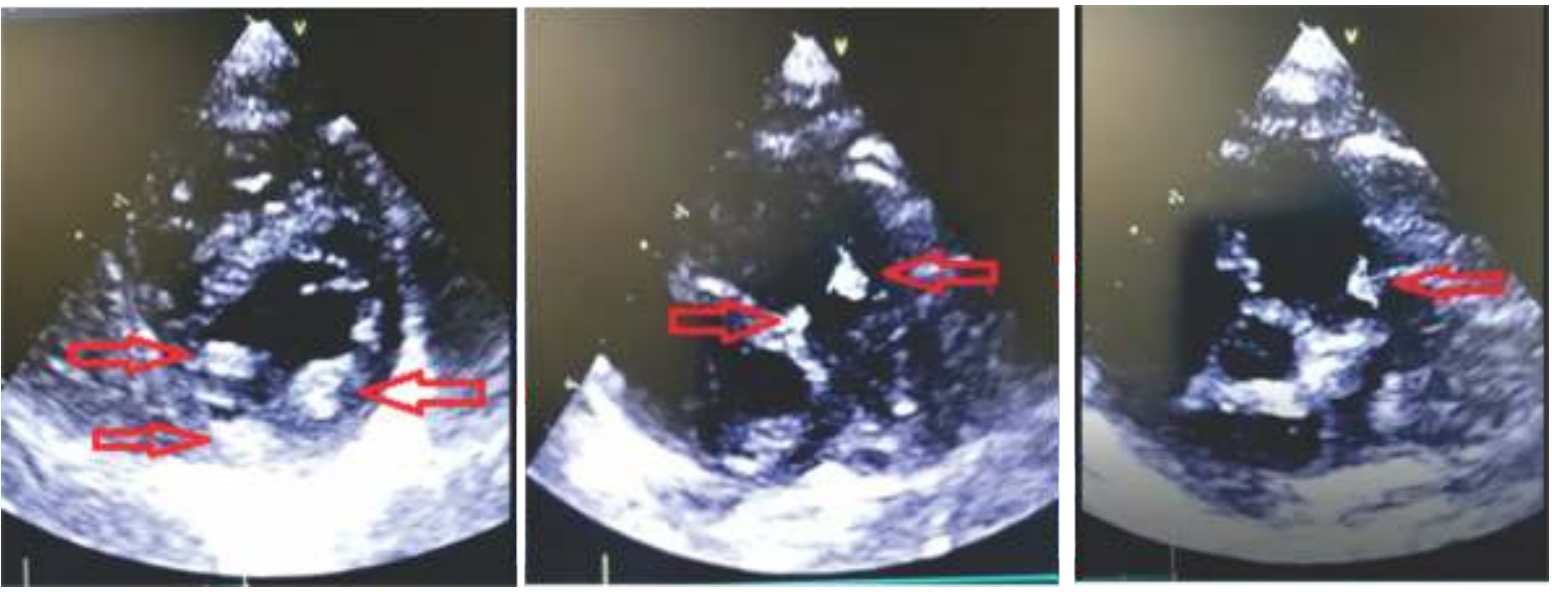

Figura 1. Ecocardiografía fetal con tumores en septum interventricular apical, otro adherido a pared posterior del ventrículo izquierdo e imagen con ecogenicidad aumentada en pared del ventrículo derecho, compatibles con túberes.

Fue hospitalizada a las 38,4 semanas, cesárea programada. Nace una recién nacida $(\mathrm{RN})$ de sexo femenino, de 3125 gramos, talla 47,2 cm, APGAR 8/9, edad gestacional por Capurro de 38.2 semanas, sin complicaciones.

La RN ingresó a sala de Neonatología para monitorización y estudios. Examen físico normal; ecografía abdominal con imagen sugerente de angiomiolipoma en polo renal inferior izquierdo
(Figura 2); la ecocardiografía neonatal confirmó hallazgos prenatales, con túberes múltiples, sin comprometer tracto de salida del ventrículo izquierdo; resonancia magnética de encéfalo (Figura 3) con nódulos sub ependimarios peri ventriculares, supratentoriales, bihemisféricos, que impresionan túberes corticales, dispersos. El examen oftalmológico reportó hamartoma periférico en región nasal superior y nasal inferior, con mácula conservada.

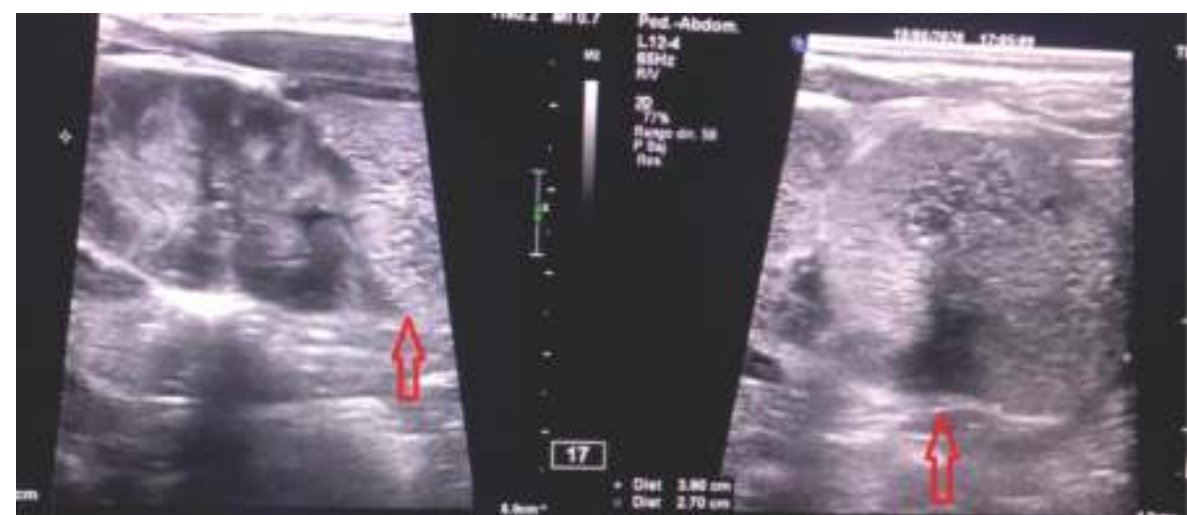

Figura 2. Ecografía abdominal: masa hiperecogénica bien delimitada, de 27 × $39 \mathrm{~mm}$, en polo renal inferior izquierdo, sugerente de angiomiolipoma. 

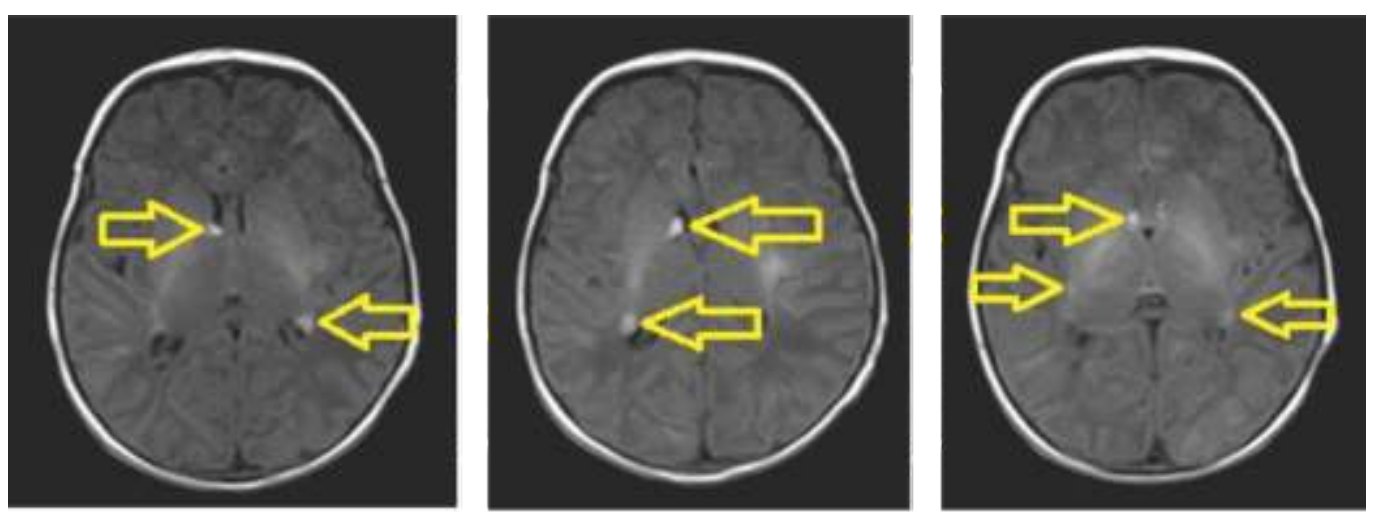

Figura 3. Resonancia magnética de encéfalo, cortes axiales en T1 con nódulos subependimarios periventriculares, bihemisféricos, espontáneamente hiperintensos.

De los criterios de Roach para el diagnóstico(3) (Tabla $1)$, esta paciente cumplió con tres mayores (angiomiolipoma renal, rabdomioma cardiaco y nódulos subependimarios) y uno menor (hamartoma retiniano). No se encontraron otras alteraciones.
La madre fue reevaluada buscando signos sugerentes, los cuales no fueron detectados. La recién nacida fue evaluada en forma multidisciplinaria y dada de alta en buenas condiciones, en planes de seguimiento cercano, con signos de alarma.

Tabla 1. Criterios mayores y menores de Roach(3) para el diagnóstico de Esclerosis tuberosa: aplicación al caso clínico.

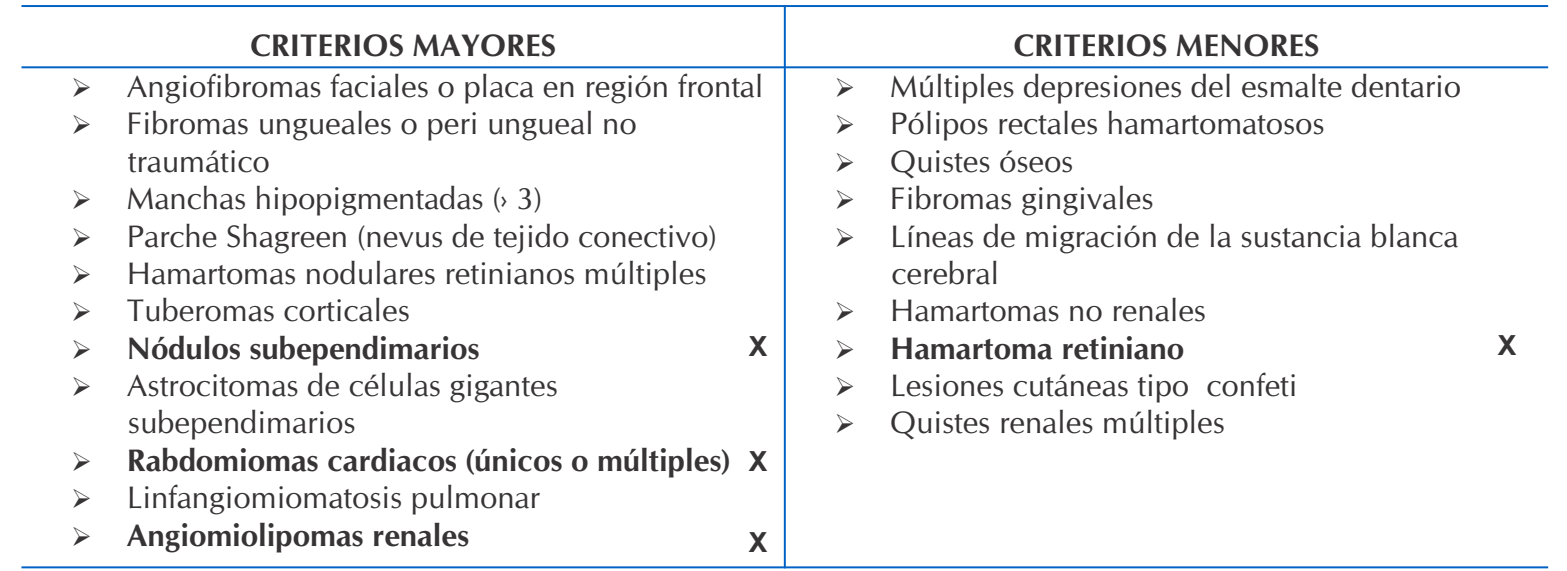

Diagnóstico definitivo: 2 criterios mayores o 1 criterios mayor y 2 criterios menores

Diagnóstico probable: 1 criterio mayor y 1 criterio menor

Diagnóstico posible: 1 criterio mayor o más de 2 criterios menores

\section{DISCUSIÓN}

El complejo esclerosis tuberosa requiere una evaluación diagnóstica que incluya historia familiar y valoración multidisciplinaria, además estudios de imagen y de funcionalidad de órganos internos dependiendo de la edad del paciente ${ }^{(4)}$.

Los rabdomiomas cardíacos suelen ser la primera manifestación clínica de la esclerosis tuberosa en el período fetal y el examen de ultrasonido en el segundo trimestre permite identificarlos ${ }^{(5)}$.

El rabdomioma es un tumor benigno que se presenta en cualquier lugar del corazón, pero es más común en el ventrículo. Las localizaciones más frecuentes 
son el ventrículo izquierdo y el tabique ventricular, aunque hasta un $30 \%$ se localizan en la pared auricular o en el ventrículo derecho ${ }^{(6)}$.

Está estrechamente relacionado con la esclerosis tuberosa y se observa en el $60 \%$ de los pacientesy los síntomas que resultan del rabdomioma cardíaco son en gran parte consecuencia del tamaño del tumor o de la ubicación dentro del corazón. La cirugía se recomienda solo para pacientes con arritmias refractarias o compromiso hemodinámico severo ${ }^{(6)}$.

En la mayoría de los casos, tiende a involucionar espontáneamente antes de los 4 años de edad y como nuestra paciente no presentaba síntomas al momento de la evaluación, se decidió control clínico y ecográfico ${ }^{(7)}$.

La resonancia magnética fetal es útil para detectar hamartomas extracardíacos, pero es difícil de utilizar como herramienta de detección ${ }^{(5)}$.

La enfermedad renal es otra de las principales causas de mortalidad en estos pacientes, por lo que se justifica la vigilancia de por vida y la intervención temprana, siendo las mujeres más vulnerables a este tipo de problemas; hallazgo constatado en nuestra paciente, en planes de seguimiento por Nefrología pediátrica $^{(8)}$.

La epilepsia es uno de los síntomas neurológicos más comunes en pacientes con esta patología, el

\section{REFERENCIAS}

1. Caylor RC, Grote L, Thiffault I, Farrow EG, Willig L, Soden $\mathrm{S}$, et al. Incidental diagnosis of tuberous sclerosis complex by exome sequencing in three families with subclinical findings. Neurogenetics. 2018;19:205-213. doi: https://doi.org/10.1007/s10048-018-0551-y

2. Almobarak S, Almuhaizea M, Abukhaled M, Alyamani S, Dabbagh O, Chedrawi A, et al. Tuberous Sclerosis Complex:Clinical Spectrum and Epilepsy: A Retrospective Chart Review Study. Transl Neurosci. 2018;9:154-160. doi: https://doi.org/10.1515/tnsci-2018-0023 estudio TOSCA mostró epilepsia en el 83,5\% de la muestra, suele comenzar durante los primeros meses de vida y, en la mayoría de los casos, antes del primer año ${ }^{(9)}$.

Los pacientes con problemas de aprendizaje tienen un riesgo significativamente mayor de mortalidad temprana $^{(8)}$ y esto implica la necesidad del diagnóstico temprano, antes del inicio de las convulsiones, fundamental para el manejo de la epilepsia y la mejoría de los resultados cognitivos ${ }^{(10)}$. Nuestra paciente no presentó crisis clínicas durante la internación, en planes de seguimiento clínico por Neurología infantil.

Las únicas manifestaciones oculares que forman parte de los criterios de diagnóstico estandarizados son las características retinianas, siendo los principales los hamartomas retinianos, presentes en aproximadamente el $50 \%$ de los individuos afectados. Estas lesiones suelen tener un impacto mínimo en la función visual, aunque se han informado casos raros de hemorragia vítrea y hamartomas retinianos agresivos que causan ceguera $^{(11)}$.

Por lo tanto, la atención médica debe ser individualizada, puntualizando el espectro clínico de la enfermedad, con el objetivo de garantizar un tratamiento oportuno, una mejor calidad de vida y poder brindar adecuado asesoramiento genético familiar $^{(4)}$.

3. Staley BA, Vail EA, Thiele EA. Tuberous sclerosis complex: diagnostic challenges, presenting symptoms, and commonly missed signs. Pediatrics. 2011;127(1):e11725. doi: https://doi.org/10.1542/peds.2010-0192

4. Nathan N, Burke K, Moss J, Darling TN. A diagnostic and management algorithm for individuals with an isolated skin finding suggestive of tuberous sclerosis complex. Br J Dermatol. 2016;176(1):220-223. doi: https://doi.org/10.1111/bjd.14724 
5. Gu X, Han L, Chen J, Wang J, Hao X, Zhang Y, et al. Antenatal screening and diagnosis of tuberous sclerosis complex by fetal echocardiography and targeted genomic sequencing. Medicine (Baltimore). 2018;97(15):e0112. doi: https://doi.org/10.1097/MD.0000000000010112

6. Kondo T, Niida Y, Mizuguchi M, Nagasaki Y, Ueno Y, Nishimura A. Autopsy case of right ventricular rhabdomyoma in tuberous sclerosis complex. Leg Med (Tokyo). 2019;36:37-40. doi: https://doi.org/10.1016/j.lega lmed.2018.10.001

7. Co-Vu J, Ivsic T. Fetal Echocardiography to Diagnose Fetal Heart Disease. NeoReviews. 2012;13(10):e590-e604. doi: https://doi.org/10.1542/neo.13-10-e590

8. Amin S, Lux A, Carder N, Laugharne M, Osborne J, O'Callaghan F. Causes of mortality in individuals with tuberous sclerosis complex. Developmental medicine \& child neurology. 2016;59(6):612-617. doi: https://onlinelibr ary.wiley.com/doi/full/10.1111/dmcn.13352

9. Nabbout R, Belousova E, Benedik MP, Carter T, Cottin V, Curatolo $\mathrm{P}$, et al. Epilepsy in tuberous sclerosis complex: Findings from the TOSCA Study. Epilepsia Open. 2018;4(1):73-84. doi: https://doi.org/10.1002/epi4.12286

10. Słowińska M, Jóźwiak S, Peron A, Borkowska J, Chmielewski D, Sadowski K, et al. Early diagnosis of tuberous sclerosis complex: a race against time. How to make the diagnosis before seizures? Orphanet J Rare Dis. 2018;13(1):25. doi: https://doi.org/10.1186/s13023-018-0764-z

11. Wan MJ, Chan KL, Jastrzembski BG, Ali A. Neuroophthalmological manifestations of tuberous sclerosis: current perspectives. Eye Brain. 2019;11:13-23. doi: https://doi.org/10.2147/EB.S186306 